\title{
Form factors and photoproduction amplitudes
}

\author{
R. M. Davidson* \\ Department of Physics, Applied Science and Astronomy \\ Rensselaer Polytechnic Institute, Troy, New York 12180-3590 \\ Ron Workman ${ }^{\dagger}$ \\ Center for Nuclear Studies and Department of Physics \\ The George Washington University Washington, DC 20052
}

(October 30, 2018)

\begin{abstract}
We examine the use of phenomenological form factors in tree level amplitudes for meson photoproduction. Two common recipes are shown to be fundamentally incorrect. An alternate form consistent with gauge invariance and crossing symmetry is proposed.
\end{abstract}

PACS numbers: 25.20.Lj, 13.60.Le, 11.40.-q, 11.80.Cr

*davidr@rpi.edu

†rworkman@gwu.edu 


\section{INTRODUCTION}

Studies of electromagnetic interactions with extended particles, such as mesons and baryons, often involve the use of (off-shell) form factors to account for internal structure not explicit in models, or to regularize quantities which would otherwise be divergent. The general structures of the $\pi \mathrm{NN}$ [1] and $\gamma \mathrm{NN}$ [2] three-point vertices allowed based on the symmetries of the strong and electromagnetic interactions have been known for decades. Of particular importance for electromagnetic interactions is gauge invariance as expressed by the Ward-Takahashi identities [3]. There is a vast literature on the subject of form factors in meson production ranging from attempts [4. 5] to satisfy the half-off-shell Ward-Takahashi identities while at the same time using as much on-shell information as possible, to explicit model calculations [6,7] which test the various prescriptions. As a rule, these model calculations demonstrate that there are kinematical regions where the prescriptions can give drastically different results than the exact model calculations.

While the most consistent way to approach this problem is through the use of fieldtheoretic models, it is not clear which field-theory approach to use in the resonance region. Although chiral perturbation theory can be used near threshold [8], it is expected to converge slowly, or not at all, at higher energies. Thus, many recent fits to meson photoproduction data have been less ambitious, and have assumed that a phenomenological approach would be adequate for the extraction of resonance contributions. These fits have generally used tree-level diagrams which require a high-energy cutoff. While the functional form of these form factors has usually been chosen on the basis of convenience and simplicity, the resulting amplitudes should obey the constraints imposed by gauge invariance, crossing and unitarity. Most efforts have been directed toward the maintenance of gauge invariance. Crossing and unitarity are often ignored in modifications applied to tree-level diagrams, whereas unitarity is generally built into dynamical models.

In the following, we will concentrate on the modification of tree-level diagrams. The effect of a minimal-substitution prescription, applied to the most general (pion-nucleon) vertex, was discussed in detail by Ohta [9] and extended by others 10 12. This work showed that a particular invariant amplitude $\left(A_{2}\right)$ would be unaltered by a wide range of form factors and meson-nucleon vertices satisfying the Ward-Takahashi [3] identity. The possibility of having a form-factor modification of $A_{2}$, accompanied by a gauge-invariance restoring contact term was subsequently considered in Ref. [10]. The recipe of Ref. [10] is simple to apply and has been widely adopted for use in single- and multi-channel fits to meson-photoproduction data 13.

We find a flaw in the arguments used to derive this modification of the amplitude $A_{2}$. It is, however, straightforward to find a modification of $A_{2}$ which does satisfy the constraints of gauge invariance and crossing symmetry. We then apply this technique to $\eta^{\prime}$ photoproduction to overcome the shortcomings of Refs. [14, 15], namely the violation of crossing symmetry. We also show how the use of our prescription can alter the conclusions of a phenomenological analysis. 


\section{REVIEW OF THE $A_{2}$ PROBLEM}

We begin by recalling the basic results given in Ref. [11]. There the discussion was simplified by taking the simplest (pseudoscalar) $\pi N N$ vertex in the specific process $\gamma p \rightarrow$ $n \pi^{+}$. The contribution from the Born diagrams corresponding to $s-, t-$, and $u$-channel exchanges is

$$
\begin{aligned}
\epsilon \cdot M_{f i} & =\sqrt{2} g e \bar{u}_{n} \gamma_{5} \frac{\left(p_{1}+k\right) \cdot \gamma+m}{s-m^{2}}\left[\epsilon \cdot \gamma-\frac{\kappa_{p}}{2 m} \epsilon \cdot \gamma k \cdot \gamma\right] u_{p} \\
& +2 \sqrt{2} g e \bar{u}_{n} \frac{q \cdot \epsilon}{t-\mu^{2}} \gamma_{5} u_{p} \\
& -\sqrt{2} g e \bar{u}_{n} \frac{\kappa_{n}}{2 m} \epsilon \cdot \gamma k \cdot \gamma \frac{\left(p_{2}-k\right) \cdot \gamma+m}{u-m^{2}} \gamma_{5} u_{p},
\end{aligned}
$$

where $k$ and $q$ represent the photon and pion four-momenta, and $p_{1}$ and $p_{2}$ are the respective proton and neutron four-momenta. The quantities $m$ and $\mu$ are the nucleon and pion masses, $\epsilon$ is the photon polarization vector, $g$ is the pseudoscalar $\pi^{0} p p$ coupling constant, and $\kappa_{p}$ and

$\kappa_{n}$ are the proton and neutron anomalous magnetic moments. Note that in Refs. [10,11], $g$ denoted the pseudoscalar $\pi^{+} n p$ coupling constant.

If a strong form factor is inserted at the $\pi N N$ vertex of each Born term, gauge invariance is clearly lost. The following term violates gauge invariance:

$$
\epsilon \cdot \bar{M}=\sqrt{2} g e \bar{u}_{n} \gamma_{5}\left[\frac{2 p_{1} \cdot \epsilon}{s-m^{2}} F\left(s, m^{2}, \mu^{2}\right)+\frac{2 q \cdot \epsilon}{t-\mu^{2}} F\left(m^{2}, m^{2}, t\right)\right] u_{p},
$$

where $F(s, u, t)$ is a general form factor. From Ohta's [9] relations, the term required to restore gauge invariance is

$\sqrt{2} g e \bar{u}_{n} \gamma_{5}\left[\frac{2 p_{1} \cdot \epsilon}{s-m^{2}}\left[F\left(m^{2}, m^{2}, \mu^{2}\right)-F\left(s, m^{2}, \mu^{2}\right)\right]+\frac{2 q \cdot \epsilon}{t-\mu^{2}}\left[F\left(m^{2}, m^{2}, \mu^{2}\right)-F\left(m^{2}, m^{2}, t\right)\right]\right] u_{p}$,

with $F\left(m^{2}, m^{2}, \mu^{2}\right)=1$. This precisely cancels the form factor effect on the terms in Eq. 2 . Writing this in terms of invariant amplitudes

$$
\epsilon \cdot M_{f i}=\bar{u}_{n} \sum_{j=1}^{4} A_{j} M_{j} u_{p}
$$

with the explicitly gauge invariant representation

$$
\begin{aligned}
& M_{1}=-\gamma_{5} \epsilon \cdot \gamma k \cdot \gamma, \\
& M_{2}=2 \gamma_{5}\left(\epsilon \cdot p_{1} k \cdot p_{2}-\epsilon \cdot p_{2} k \cdot p_{1}\right), \\
& M_{3}=\gamma_{5}\left(\epsilon \cdot \gamma k \cdot p_{1}-\epsilon \cdot p_{1} k \cdot \gamma\right), \\
& M_{4}=\gamma_{5}\left(\epsilon \cdot \gamma k \cdot p_{2}-\epsilon \cdot p_{2} k \cdot \gamma\right),
\end{aligned}
$$

noting that such a representation is only possible for a gauge invariant amplitude, we have 


$$
\begin{aligned}
& A_{1}=\frac{\sqrt{2} g e F\left(s, m^{2}, \mu^{2}\right)}{s-m^{2}}\left(1+\kappa_{p}\right)+\frac{\sqrt{2} g e F\left(m^{2}, u, \mu^{2}\right)}{u-m^{2}} \kappa_{n}, \\
& A_{2}=\frac{2 \sqrt{2} g e}{\left(s-m^{2}\right)\left(t-\mu^{2}\right)}, \\
& A_{3}=\frac{\sqrt{2} g e F\left(s, m^{2}, \mu^{2}\right)}{s-m^{2}} \frac{\kappa_{p}}{m}, \\
& A_{4}=\frac{\sqrt{2} g e F\left(m^{2}, u, \mu^{2}\right)}{u-m^{2}} \frac{\kappa_{n}}{m} .
\end{aligned}
$$

Thus, one sees that the $A_{2}$ amplitude is unaltered by form factors, within Ohta's prescription for retaining gauge invariance. The result holds for a more general pion-nucleon vertex and any charge channel. Of course, this result does not prevent the addition of terms which are individually gauge invariant.

Since form factors were added to provide a cutoff for the Born term contributions, this result is somewhat disappointing, as the $A_{2}$ contribution is not damped. In addition, explicit one-loop meson calculations [7,8] show that the $A_{2}$ amplitude is modified. In Ref. [10], a form factor for $A_{2}$ was inserted and the addition of a contact interaction was proposed to restore gauge invariance. We give this recipe below, show why it has a problem, and then propose an alternate form. The result is then generalized using crossing symmetry.

\section{THE HABERZETTL FORM FACTOR}

In Ref. [10] a gauge invariant term, $\hat{F} A_{2}$, was added and subtracted from a set of Born terms with form factors at the strong vertices. This resulted in the replacement $A_{2} \rightarrow \hat{F} A_{2}$ above, with a remaining gauge-invariance-violating term

$$
\begin{aligned}
\epsilon \cdot M_{\text {viol }} & =\sqrt{2} g e \bar{u}_{n} \gamma_{5}\left[\frac{2 p_{1} \cdot \epsilon}{s-m^{2}}\left(F\left(s, m^{2}, \mu^{2}\right)-\hat{F}\right)\right. \\
& \left.+\frac{2 q \cdot \epsilon}{t-\mu^{2}}\left(F\left(m^{2}, m^{2}, t\right)-\hat{F}\right)\right] u_{p}
\end{aligned}
$$

which was to be cancelled by a contact interaction.

By requiring this additional term to be free of poles, i.e., a contact term, we can constrain the functional form of $\hat{F}$. Denoting $F\left(s, \mu^{2}, m^{2}\right) \equiv F_{1}(s)$, with similar abbreviations for $F_{2}(u)$ and $F_{3}(t)$, subject to the constraints

$$
F_{1}\left(m^{2}\right)=F_{2}\left(m^{2}\right)=F_{3}\left(\mu^{2}\right)=1,
$$

the general form for $\hat{F}$ used in Ref. [10] was

$$
\hat{F}=a_{1} F_{1}(s)+a_{2} F_{2}(u)+a_{3} F_{3}(t),
$$

subject to $a_{1}+a_{2}+a_{3}=1$. This form has been used in many recent analyses of photonic and multi-channel reactions off the nucleon [13]. However, it is straightforward to demonstrate that this recipe results in a term with pole contributions, and is therefore incorrect because it cannot be cancelled by a contact term. Taking $a_{2}=1$, and inserting $\hat{F}$ into the relation 
for $M_{\text {viol }}$, one can easily see the problem. Choosing, for example, the point $s=m^{2}$ does not fix the value of $u$, which is only constrained by the condition $t+u=\mu^{2}+m^{2}$. As a consequence, when $s=m^{2}$ it is not necessarily the case that $F_{2}(u)=1$. As a result, the pole terms are not cancelled, except for zero photon energy, as noted by the authors. To ensure cancellation of the poles, the needed constraint is, in this case,

$$
\hat{F}\left(s=m^{2}, t\right)=\hat{F}\left(s, t=\mu^{2}\right)=1
$$

which does not, however, imply that $\hat{F}(s, t)=1$ for all $s$ and $t$. For example, the following choice円

$$
\hat{F}=F_{1}(s)+F_{3}(t)-F_{1}(s) F_{3}(t)
$$

satisfies the above constraint. Note that this method can be easily adapted to charged kaon production.

So far, we have only considered constraints provided by gauge invariance and the pole structure of the amplitude. However, crossing symmetry is also important. As the $p \pi^{-}$ channel is closely related to the $n \pi^{+}$channel via crossing, let us therefore consider the isospin channel $T^{(-)}$defined by

$$
T^{(-)}=\frac{1}{2 \sqrt{2}}\left(T^{n \pi^{+}}-T^{p \pi^{-}}\right)
$$

Inserting strong form factors, the matrix element for the electric Born terms is

$$
\begin{aligned}
\epsilon \cdot M & =\frac{e g}{2} F_{1}(s) \bar{u}_{2} \gamma_{5} \frac{\left(p_{1}+k\right) \cdot \gamma+m}{s-m^{2}} \gamma \cdot \epsilon u_{1} \\
& -\frac{e g}{2} F_{2}(u) \bar{u}_{2} \gamma \cdot \epsilon \frac{\left(p_{2}-k\right) \cdot \gamma+m}{u-m^{2}} \gamma_{5} u_{1} \\
& +2 e g F_{3}(t) \bar{u}_{2} \gamma_{5} \frac{q \cdot \epsilon}{t-\mu^{2}} u_{1} .
\end{aligned}
$$

After some rearrangement, this may be written as

$$
\begin{aligned}
\epsilon \cdot M & =\frac{e g}{2} \bar{u}_{2} M_{1}\left[\frac{F_{1}(s)}{s-m^{2}}-\frac{F_{2}(u)}{u-m^{2}}\right] u_{1} \\
& +e g \hat{F}(s, u, t) \bar{u}_{2} \frac{M_{2}}{\left(t-\mu^{2}\right)}\left[\frac{1}{s-m^{2}}-\frac{1}{u-m^{2}}\right] u_{1} \\
& +\epsilon \cdot \Delta M
\end{aligned}
$$

where $M_{1,2}$ are the gauge invariant operators defined in Eq. 5 and

\footnotetext{
${ }^{1}$ It should be noted that this choice is not unique.

${ }^{2}$ The isoscalar channel, $T^{(0)}$, is discussed below.
} 


$$
\begin{aligned}
\epsilon \cdot \Delta M & =-\frac{e g}{2} \bar{u}_{2} \gamma_{5}\left[\frac{2 p_{1} \cdot \epsilon}{s-m^{2}}\left(\hat{F}(s, u, t)-F_{1}(s)\right)\right. \\
& \left.+\frac{4 q \cdot \epsilon}{t-\mu^{2}}\left(\hat{F}(s, u, t)-F_{3}(t)\right)-\frac{2 p_{2} \cdot \epsilon}{u-m^{2}}\left(\hat{F}(s, u, t)-F_{2}(u)\right)\right] u_{1} .
\end{aligned}
$$

As $A_{1,2}^{(-)}$are odd under crossing, i.e.,

$$
A_{1,2}^{(-)}(s, u, t)=-A_{1,2}^{(-)}(u, s, t)
$$

we see that $\hat{F}(s, u, t)=\hat{F}(u, s, t)$ and $F_{2}(u)=F_{1}(u)$, that is, $F_{2}(u)$ is obtained from $F_{1}(s)$ by the replacement $s \rightarrow u$. In order to cancel the poles in $\epsilon \cdot \Delta M$, so this term may be cancelled by a contact term, we need

$$
\hat{F}\left(m^{2}, u, t\right)=\hat{F}\left(s, m^{2}, t\right)=\hat{F}\left(s, u, \mu^{2}\right)=1 .
$$

Although not unique, one crossing symmetric form for $\hat{F}$ that satisfies the above constraints is

$$
\begin{aligned}
\hat{F}(s, u, t) & =F_{1}(s)+F_{1}(u)+F_{3}(t) \\
& -F_{1}(s) F_{1}(u)-F_{1}(s) F_{3}(t)-F_{1}(u) F_{3}(t)+F_{1}(s) F_{1}(u) F_{3}(t) .
\end{aligned}
$$

\section{PHENOMENOLOGY}

The use of different form factor schemes can have a significant effect on phenomenological fits to data. This is particularly clear in kaon photoproduction where the rapid increase of a point-like Born contribution to this quantity is well known. The point-like Born term contribution to the total cross section (solid) is compared to the data [16] in Fig. 1 using a $K \Lambda N$ coupling of 7.5 [17]. An attempt to cure this, by modifying individual multipoles, was described in Ref. [18]. Using the minimal substitution prescription of Ohta [9] (dotted), which damps all but the $A_{2}$ amplitude, one still obtains a Born contribution which grows too rapidly. Here, we use

$$
F_{1}(s)=\left[1+\left(s-m^{2}\right)^{2} / \Lambda^{4}\right]^{-1},
$$

with a cutoff mass $\Lambda$ of $1 \mathrm{GeV}$. Finally, we show in Fig. 1 the result arising from our modification of the Haberzettl form (dashed). In addition to $F_{1}(s)$ given above, we also need $\hat{F}$ and $F_{3}(t)$. We take $\hat{F}$ to be of the form of Eq. (11) with

$$
F_{3}(t)=\left[1+\left(t-\mu^{2}\right)^{2} / \Lambda^{4}\right]^{-1},
$$

where $\mu$ is the kaon mass and we again take $\Lambda=1 \mathrm{GeV}$.

Finally, we consider the fits to $\eta^{\prime}$ photoproduction performed in Refs. [14, 15]. In these studies, the Born terms were added and compared to possible resonance contributions. The use of a coupling, $g_{\eta^{\prime}}$, within the range of values, $1 \leq g_{\eta^{\prime}} \leq 6$, estimated in this work [14] results in an (undamped) Born contribution exceeding the data 19 21 by a large factor, as is demonstrated by the solid line in Fig. 2 (here, $g_{\eta^{\prime}}=1.9$ ). There is more than one 
way to interpret this result. One possibility is that $g_{\eta^{\prime}}$ is much smaller than estimated in Ref. [14]. A different possibility is that $g_{\eta^{\prime}}$ is in the range given above, but the effective $g_{\eta^{\prime}}$ is much smaller than $g_{\eta^{\prime}}$ at the nucleon pole. In other words, as the proton and eta-prime are extended objects, form factors enter at the three-point vertices, and as these data are far from the nucleon pole, form factor effects can be significant. Finally, there are almost certainly other reaction mechanisms such as resonance exchanges, vector meson exchanges, and possibly contact terms.

In the original analysis of the $\eta^{\prime}$ data [14, the Born terms were multiplied by an overall form factor given by Eq. ([19) with $\Lambda=1.1 \mathrm{GeV}$. In this model, the dominant contribution to the model was from the background of the D13(2080) resonance, and the Born terms played a minor role. The resulting total cross section from the Born terms is shown by the dashed (nearly zero) line in Fig. 2. On the other hand, taking $g_{\eta^{\prime}}=3.5$ and $\Lambda=2 \mathrm{GeV}$, it was shown that one could qualitatively reproduce the total cross section with just Born terms (dotted line) [15]. However, the procedure of multiplying the Born terms by an overall form factor violates crossing symmetry and the amplitude has the wrong residue structure, and therefore is theoretically unacceptable. Other inconsistencies of this procedure have been discussed in Refs. [6, [7]. Thus, it is not clear what conclusions, if any, can be drawn from the dashed and dotted curves in Fig. 2.

Using Ohta's [9] method, one obtains a crossing symmetric amplitude, but as above the $A_{2}$ amplitude is not modified. Using our modification of the Haberzettl form, the electric Born terms are

$$
\begin{aligned}
& A_{1}=e g_{\eta^{\prime}}\left[\frac{F_{1}(s)}{s-m^{2}}+\frac{F_{1}(u)}{u-m^{2}}\right] \\
& A_{2}=\frac{2 e g_{\eta^{\prime}}}{\left(s-m^{2}\right)\left(u-m^{2}\right)} \hat{F}(s, u),
\end{aligned}
$$

where $F_{1}\left(m^{2}\right)=1$. In this case, the constraints on $\hat{F}(s, u)$ are

$$
\hat{F}\left(s, m^{2}\right)=\hat{F}\left(m^{2}, u\right)=1,
$$

from the condition to remove the poles, and $\hat{F}(s, u)=\hat{F}(u, s)$ from crossing symmetry. Note that this form is also valid for $\pi^{0}$ and $\eta$ production, and the electric Born terms for isoscalar production of pions, defined by

$$
T^{(0)}=\frac{1}{2 \sqrt{2}}\left(T^{n \pi^{+}}+T^{p \pi^{-}}\right),
$$

can be obtained from Eq. (21) using the replacement $g_{\eta}^{\prime} \rightarrow g / 2$. With this form, the Born terms in $\eta^{\prime}$ production can be sufficiently damped at high energies even for the largest estimated value of $g_{\eta}^{\prime}$. For example, taking

$$
\hat{F}=F_{1}(s)+F_{1}(u)-F_{1}(s) F_{1}(u),
$$

with $F_{1}(s)$ given by Eq. (19), and using the upper limit for $g_{\eta^{\prime}}$ with a cutoff of $700 \mathrm{MeV}$, the total cross section from the Born terms levels off at about $0.2 \mu \mathrm{b}$ at a photon energy of $2 \mathrm{GeV}$. Furthermore, and this is the essential point, it is not possible to reproduce the 
total cross section shape here with only Born terms and reasonable values for $\Lambda$ (500 to $2000 \mathrm{MeV}$ ), in contrast to the non-crossing symmetry model of Ref. [15]. Thus, the use of the present, more restrictive, form factor prescription narrows the possible interpretations of experimental data.

\section{CONNECTION WITH CONTACT TERMS}

It is known in field theory [22] that off-shell form factors are not unique and are closely related to contact terms. For different representations of the fields, the form factors and contact terms differ, but the differences cancel out in the calculation of a physical scattering amplitude. In the case of dispersion relations [1,2], the nonuniqueness of the off-shell form factors is reflected in the a priori unknown number of needed subtractions [23]. For the phenomenological amplitudes presented here, it is also possible to shift form factor effects to contact terms. To illustrate this, recall that $F_{1}\left(m^{2}\right)=1$ and we may write, for example, for the $A_{1}$ term in Eq. (21),

$$
A_{1}=e g_{\eta^{\prime}}\left[\frac{1}{s-m^{2}}+\frac{1}{u-m^{2}}+\frac{\left(F_{1}(s)-1\right)}{s-m^{2}}+\frac{\left(F_{1}(u)-1\right)}{u-m^{2}}\right] \text {. }
$$

The first two terms above are recognized as the undamped Born terms, while the last two terms correspond to contact terms since the pole is cancelled as $s$ or $u \rightarrow m^{2}$. As previously noted, the $A_{2}$ amplitude is not modified in Ohta's method [9], but his method does not forbid the inclusion of an additional gauge invariant contact term. Our modification of Haberzettl's procedure is equivalent to adding the gauge invariant contact term,

$$
A_{2} \bar{u}_{2} M_{2} u_{1}(\hat{F}-1)
$$

to the amplitude of Ref. [11] (Eq. (6) of this work). Here, $M_{2}$ is the gauge invariant operator defined in Eq. (5) and $A_{2}$ is given by the second equation of Eqs. (6).

Finally, let us note that most authors assume that the form factors are real, and the phenomenological results presented here are also obtained using real form factors. In general, however, arguments based on unitarity [1,2,23] show that the off-shell form factors must be complex above the one-pion threshold. On the other hand, there is some justification for using real form factors if one adopts a K-matrix approach to the scattering amplitude [24].

\section{CONCLUSIONS}

We have found a flaw in the gauging procedure of Ref. [10] and have noted that the method used in Refs. [14,15] is theoretically unacceptable as it violates crossing symmetry.

We have determined the general constraints $\hat{F}$ must satisfy for the gauge-violating term to be a contact term, which then can be cancelled by a contact term. This method was generalized to be consistent with crossing symmetry and applied to $\eta^{\prime}$ photoproduction. This model is theoretically superior to previous works [14,15] and has the important phenomenological consequence that the total cross section cannot be interpreted as arising solely from the Born terms, in contrast to Ref. [15]. Other phenomenological applications are being investigated. 


\section{ACKNOWLEDGMENTS}

We are grateful to Nimai C. Mukhopadhyay for many useful discussions regarding etaprime photoproduction and the need for a crossing symmetric model. We thank Paul Stoler for a critical reading of the manuscript. This work was supported in part by the U. S. Department of Energy Grants DE-FG02-99ER41110 and DE-FG02-88ER40448. R.W. gratefully acknowledges a contract from Jefferson Lab under which this work was done. Jefferson Lab is operated by the Southeastern Universities Research Association under the U. S. Department of Energy Contract DE-AC05-84ER40150. R.M.D. thanks the Center for Nuclear Studies for supporting a visit in which part of this work was completed. 
FIG. 1. The total cross section for $\gamma p \rightarrow K^{+} \Lambda$ versus the photon lab-energy, $E_{\gamma}$. Plotted are the unmodified Born terms (solid), Ohta's prescription [9] (dotted), and our form-factor modification (see text). Data from Ref. [16].

FIG. 2. The total cross section for $\gamma p \rightarrow p \eta^{\prime}$ versus the photon lab-energy, $E_{\gamma}$. The data are from SAPHIR [21] (diamonds), AHHM [20] (squares) and ABBHHM 19] (crosses). The solid line is the total cross section obtained from the Born terms with $g_{\eta^{\prime}}=1.9$ and without any form factors. The dashed line is from the work of Ref. [14] where the Born terms are multiplied by an overall form factor depending only on $s$, thus violating crossing symmetry. The dotted line is the best Born model fit to the data using the non-crossing symmetric model [15].

\section{REFERENCES}

[1] E. Kazes, Nuov. Cim. 13, 1226 (1959).

[2] A. Bincer, Phys. Rev. 118, 855 (1960).

[3] J.C. Ward, Phys. Rev. 78, 182 (1950); Y. Takahashi, Nuovo Cimento 6, 371 (1957).

[4] F.A. Berends and G.B. West, Phys. Rev. 188, 2538 (1969).

[5] F. Gross and D. Riska, Phys. Rev. C 36, 1928 (1987).

[6] H.W.L. Naus and J.H. Koch, Phys. Rev. C 39, 1907 (1989).

[7] J.W. Bos, S. Scherer and J.H. Koch, Nucl. Phys. A547, 488 (1992).

[8] V. Bernard, N. Kaiser, and U.-G. Meissner, Phys. Lett. B282, 448 (1992); ibid, B378, 337 (1996).

[9] K. Ohta, Phys. Rev. C 40, 1335 (1989).

[10] H. Haberzettl, C. Bennhold, T. Mart, and T. Feuster, Phys. Rev. C 58, R40 (1998).

[11] R.L. Workman, H.W.L. Naus, and S.J. Pollock, Phys. Rev. C 45, 2511 (1992).

[12] S. Wang and M.K. Banerjee, Phys. Rev. C 54, 2883 (1996).

[13] T. Feuster and U. Mosel, Phys. Rev. C 59, 460 (1999); T. Mart and C. Bennhold, Phys. Rev. C 61, 012201(R) (1999); T. Mart, Phys. Rev. C 62, 038201 (2000); F.X. Lee, T. Mart, C. Bennhold, H. Haberzettl, L.E. Wright, nucl-th/9907119.

[14] J.-F. Zhang, N.C. Mukhopadhyay and M. Benmerrouche, Phys. Rev. C 52, 1134 (1995).

[15] N.C. Mukhopadhyay, J.-F. Zhang, R.M. Davidson and M. Benmerrouche, Phys. Lett. B410, 73 (1997).

[16] Data from the SAID database (http://gwdac.phys.gwu.edu). This source also contains representative fits from several different groups.

[17] This value for the $K \Lambda N$ coupling is within the range of values found or used in: R.A. Adelseck, C. Bennhold, and L.E. Wright, Phys. Rev. C 32, 1681 (1985) and Ref. [10].

[18] H. Tanabe, M. Kohno and C. Bennhold, Phys. Rev. C 39, 741 (1989).

[19] The ABBHHM collaboration, Phys. Rev. 175, 1669 (1968).

[20] The AHHM collaboration, Nucl. Phys. B108, 45 (1976).

[21] R. Plötzke et al., Phys. Lett. B444, 555 (1998).

[22] S. Scherer and H. Fearing, Phys. Rev. C 51, 359 (1995).

[23] R.M. Davidson and G.I. Poulis, Phys. Rev. D 54, 2228 (1996).

[24] S. Kondratyuk and O. Scholten, Phys. Rev. C 59, 1070 (1999). 


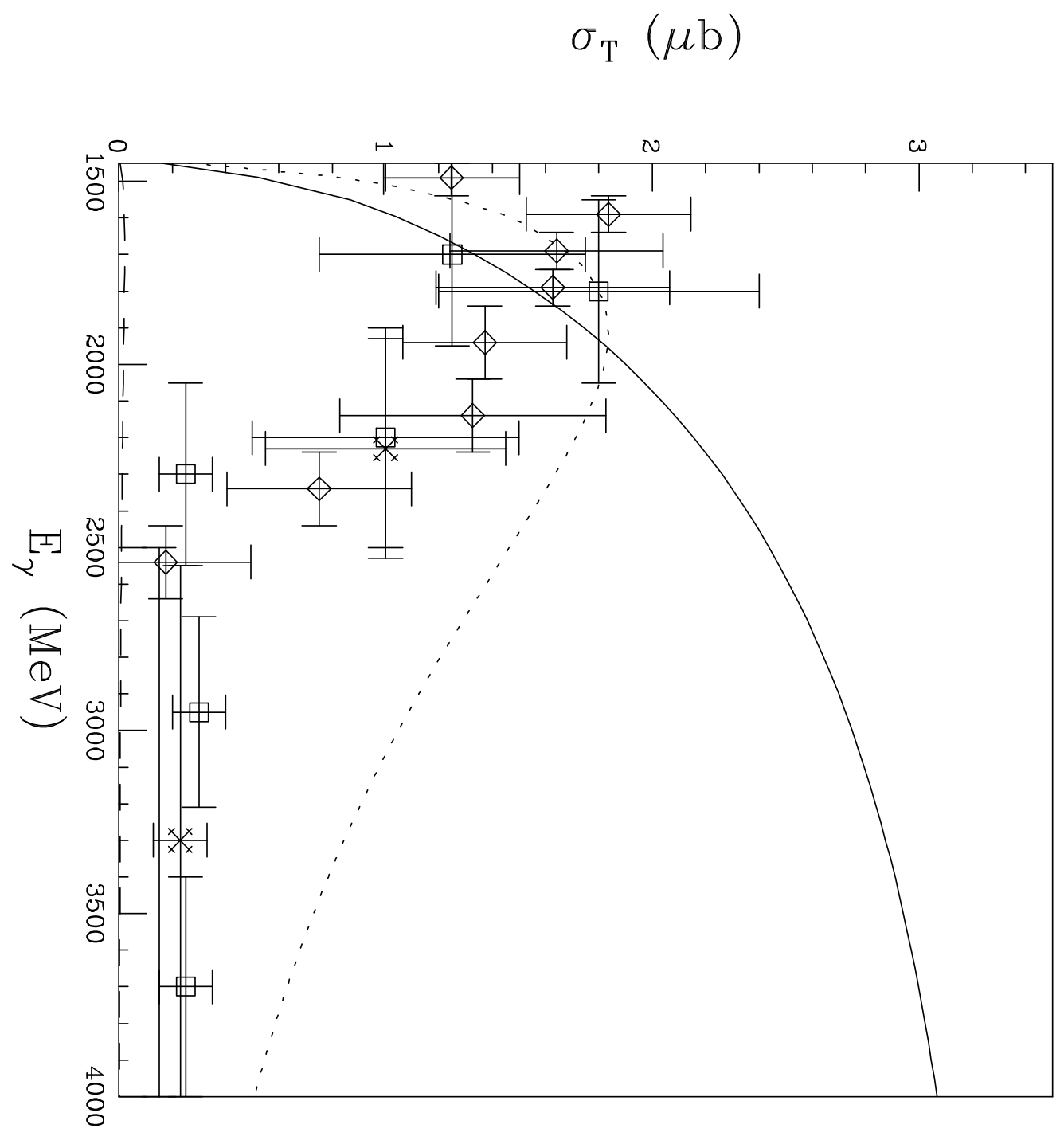




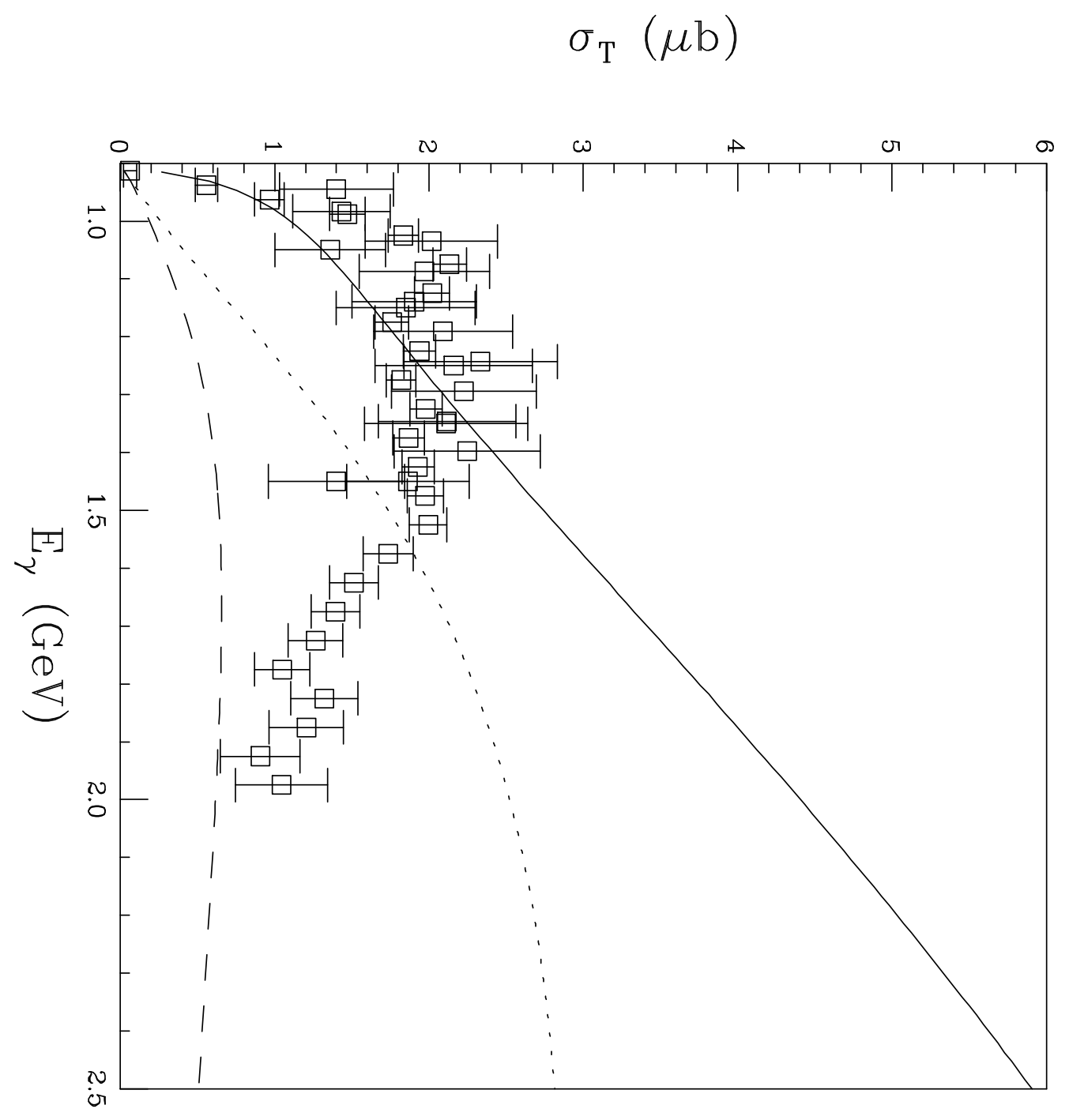

